$$
19.1): 12(47) 1--4
$$

LA-UR- 92-985

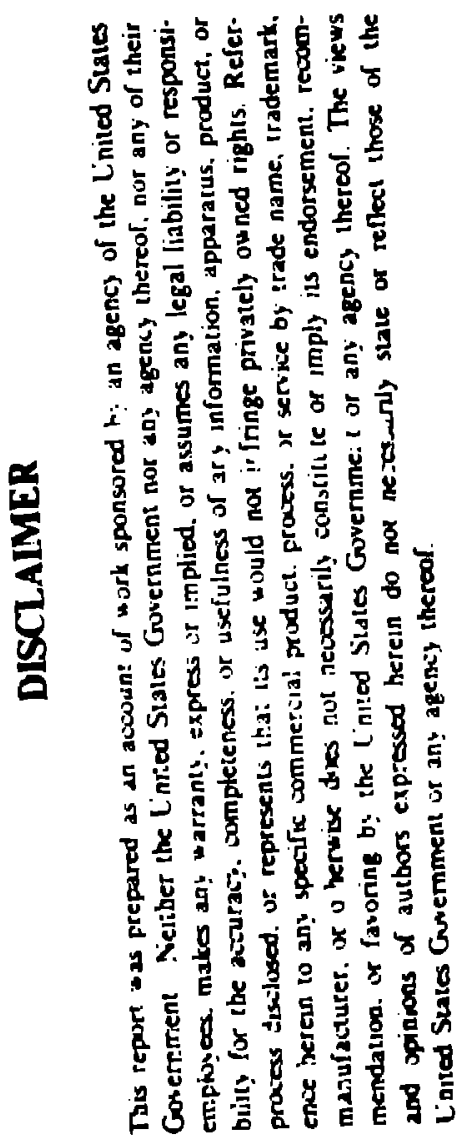

Author(s):

Submitfed to

Mdjacency-Blurring-Effect of Scenes Modeled by the Radiosity Method

LA-UR- $-92-985$

DE92 011496

C.C. Borel and S.A.W. Cerstl

sucioty of photo-ontical Instrumentation Enoinerers, (Irlando. Fi, 20-24 April 1992.

Pon

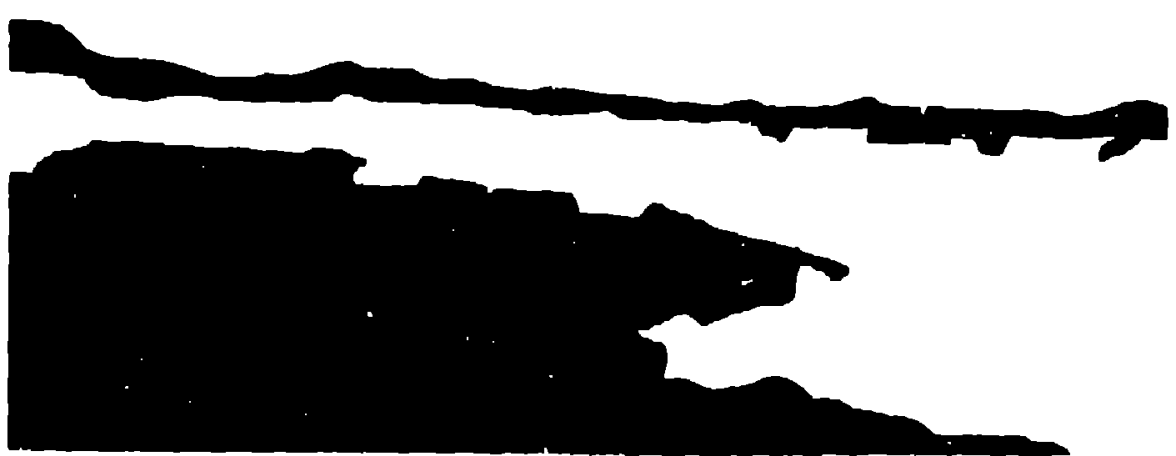

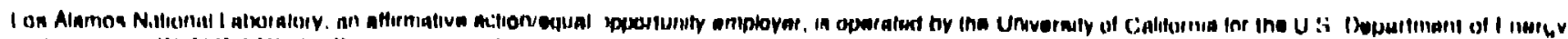

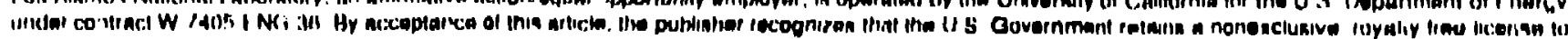

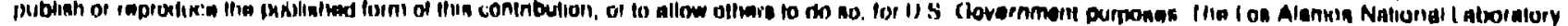

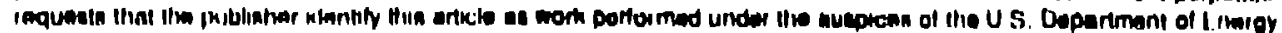




\title{
ADJACENCY-BLURRING-EFFECT OF SCENES MODELED BY THE RADIOSITY METHOD
}

\author{
C.C. Borel and S.A.W. Gerstl \\ Space Science \& Technology, SST-8, MS D438 \\ Los Alamos National L aboratory \\ I.os Alamos, New Mexico 87545, USA
}

\begin{abstract}
In this paper we describe a method to simulate images through a scattering atmosphere. We compute the scattering of light from adjacent surfaces into the ficld-of-view (FOV) with the extended radiosity method. Our simulation takes aerosol scattering phase functions and ground bidirectional reflectance distributions (BRDF) into account.
\end{abstract}

\section{Introduction}

The adjacency-blurring-effect is observed at the boundary between a dark and a bright surface. Near the edge uver a dark surface photons from the nearby bright surface may be scattered within the atmosphere into the field of view (FOV) of an airborne or satellite sensor. Conversely, near the edge over a bright surface fewer photons reach the sensor's FOV. At a sharp discontinuity in the surface reflectance the intensity transect appears as a sigmaoid instead of a step function.

The adjacency-bl-irring-effect may introduce errors in the detection and classification of smali bright targets qurr,. ' ed by a dark region or dark targets on a bright background. One can inodel the blurring due (o) he adjacency effect with a point spread function (PSF). This PSF is a filter function which is $(0,0$ volved with the unperturbed (no almosphere) refiectance image of a surface. Other researchers of uhe adjacency effect are: Pearce (1977), T'anré (1981), Kaufman (1984), Diner and Martonchik (1985) and Richter (1990). Most PSP's are generated by Monte Carlo based methods and are assumed to be rotationally symmetric, thus are not valid for off-nadir views. In the siluation of an oblique viewing sensor it is necessary to compute off-nadir PSF's which are geierally asymmetric:

W' introduce a method to compute the point spread function for any view direction and any layered at mosphere: which includes scaltering phase functions. The method is based on the extended radiosity 'nethod, Borel and (ierstl (1991). We then refine the method to non-l,ambertian surfaces and show an algo:ithm to simulate adjarency blurred images which incliede vegetated, hare soil and water surfacies.

\section{Compnting tho Point Spread Function for Lambertian Surfaces}

In an optical system the poini spread function $P S P\left(x, y, z ; r_{0}, y_{1}, z_{0} ; \theta_{0}, \phi_{a} ; \theta_{r}, \phi_{r}\right)$ (anl he defined as the scattering contribution of a surface element dd ds dy illuminated from direction $\left(\theta_{0}, \phi_{a}\right)$ localed at $\left(s_{1}, y_{,} z \quad z_{i}\right)$ into the line-of sight direction of the observer $\left(\theta_{r}, \phi_{r}\right)$ lonking at point $\left(x_{11}, y_{11}, z_{1}\right)$. lighure I showe the peometry for the ground to ntmosphere scallering. (Bne can show 
(see Borel and Gerstl (1992)) that the unitless PSF is given by :

$$
P S F(x, y, \ldots)=\frac{\kappa, \Delta l}{4 \pi} \sum_{k=1}^{K} \frac{\tau\left(r_{k}\right) \cos \theta_{r, k} f\left(\theta_{p, k}\right) d x d y}{\pi r_{k}^{2}} \cdot \exp \left(-\kappa_{t}(K-k) \Delta l\right),
$$

where $\kappa_{2}$ is the scat tering coefficient in $\left[m^{-1}\right], \Delta l=L_{z} /\left(K \cos \theta_{r}\right), L_{z}$ is the height of the scattering atmosphere, $K$ is the number of layers in the atmosphere, $\tau\left(r_{k}\right)=\exp \left(-\kappa_{t} \tau_{k}\right), \kappa_{t}$ is the total scattering coefficient in $\left[m^{-1}\right], r_{k}$ is the distance between surface point $\vec{P}$ and a point $\vec{P}_{k}$ on the line-of-sight in the $k$-th layer, $\theta_{r, k}$ the view zenith angle to $d A, f\left(\theta_{p, k}\right)$ is the scat tering phase function of the $k$-th layer and $\theta_{p, k}$ is the scattering phase angle. Note that this method takes height dependent scattering and absorption coefficients and even height dependent scattering phase functions into account. The method can even be extended to include terrain effects when the PSF is coinputed for each pixel in the scene. For the Lambertian surface the PSF for nadir view is rotationally symmetric and asymmetric for all non-nadir views.

According to Borel and Gerstl (1992), the measured radiance $I_{\text {measured }}\left(x, y, z ; x_{0}, y_{0}, z_{0} ; \theta_{0}, \phi_{a i}\right.$ $\left.\theta_{r}, \phi_{r}\right)$ in $\left[W m^{-2}\right]$ at the sensor for a Lambertian surface is given by :

$$
I_{\text {meaured }}(x, y, \ldots)=\frac{E_{0}}{\pi} T_{-}\left[T_{r} \rho\left(x_{0}, y_{0}\right)+\rho(x, y) \otimes P S F(x, y, \ldots)\right]+I_{\text {path }}
$$

where $E_{0}$ is the direct energy incident from the sun in $\left[W m^{-2} \mid, \tau_{d}=\exp \left(-\kappa_{\mathrm{l}} L_{2} / \cos \theta_{\mathrm{l}}\right), \tau_{\mathrm{r}}=\right.$ $\exp \left(-\kappa_{t} L_{s} / \cos \theta_{r}\right), \rho(x, y)$ is the reflectance at point $(x, y), \theta$ denotes the convolution and $I_{\text {path }}$ is the path radiance or radiance due to scattering in the at mosphere.

\section{The Point Spread Function for Non-Lambertian Surfaces}

Ir. practical situations the imaged ground surface is non-Lambertian and thus the above described method may lead to inaccurate simulated innages. We attempl now to include the bidirectional reflectance distribution function (BRDF) in the compulation of the PSF. First, let us assuine that the entire surface has the BRDF :

$$
f\left(x, y, z ; \theta_{n}, \phi_{2} ; \theta_{r} ; \phi_{r}\right)-f\left(\theta_{n}, \phi_{0} ; \theta_{r}, \phi_{r}\right) .
$$

Second, that the contributions from indirect skylight are negligible on the radiance in direction $\left(\theta_{r, k}, \phi_{r, k}\right)$ or that the upwelling radiance $I_{\text {ground }}$ at the ground level is proportional to $f\left(\theta_{2}, \phi_{2} ; \theta_{r}, \phi_{r}\right)$. 'This sccond assumption clearly is not valiel for turbid atmospheres and for highly sperular surfaces like water. Under these two assumptions we can replace $\rho(x, y)$ in eq $(1)$ with $\pi f\left(\theta_{n}, \phi_{a} ; \theta_{r}, \phi_{r}\right)$.

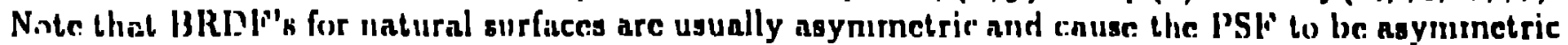
for all view directions, including nadir. 'The PSF for non-lasmbertian surfueces is then given by :

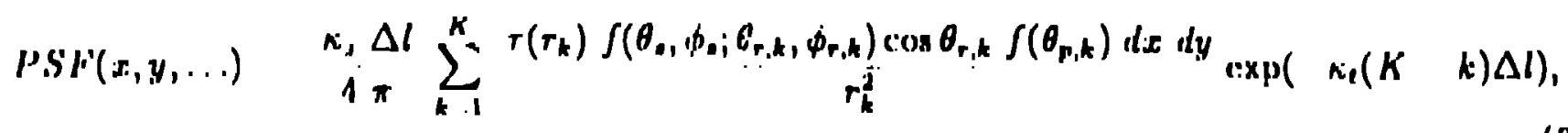

where $\phi_{r, k}$ in the view nzimuth nngle of surface dA from point $l_{k}$.

\section{Simulation of Se:enes with Iteterogencenss Surface Cover}

The liarthe, surface is eomposed of a mosnic: of various surface typen such as vepetation, bare soil

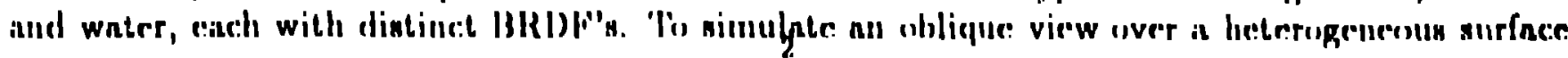


the following algorithnı was used :

1. For each ourface BRDF $f_{1}\left(\theta_{n}, \phi_{s} ; \theta_{r}, \phi_{r}\right), i=1,2, \ldots, N$ compute the point spread function $P S F_{i}(x, y, \ldots)$ using eq (3j).

2. Generate a binary image $Q_{i}(x, y)$ for each surface type 2 , where $Q_{b}(x, y)=1$ if the point $(x, y)$ has surface cover type $i$ and 0 other wise.

3. Convolve each image $Q_{i}(x, y)$ with its point spread function $P S F_{i}(x, y, \ldots)$.

The measured radiance image is then given by :

$$
I_{\text {measured }}(x, y)=\frac{E_{0}}{\pi} \tau_{1} \sum_{i=1}^{N}\left[\tau_{r} Q_{1}\left(x_{0}, y_{0}\right) f_{i}\left(\theta_{a}, \phi_{a} ; \theta_{r}, \phi_{r}\right)+Q_{i}(x, y) \leftrightarrow P S F_{i}(x, y, \ldots)\right]+I_{\text {path }}
$$

Equation (4) shows that the adjacency blurring effert is the arperposition of ground cover type images convolved with their corresponding point spread functions.

To illustrate the method on an example we used scattering phase function and BRDF's found in the literature.

To approximate a "hazy" atmosphere the Henyey-Greenstein phase function wo.r uged with the asymmetry factor $\Theta=0.75$ (Liou (1980)). The llenyey-Greenstein phase function is defined as :

$$
f\left(\theta_{p}\right)=\frac{1-\theta^{2}}{\left(\overline{1}+\theta^{2}-2 \theta \cos \partial_{p}\right)^{3 / 2}} .
$$

We selected a $\kappa_{t}=0.8, \kappa_{a}=0.05$ for an acrosol laden atmosphere of $1000 \pi_{t}$ height with 20 layers and a surface of $3000 \mathrm{~m}$ by $3000 \mathrm{~m}$ horizontal cxtent with 30 by 30 pixels.

The BRDF of bare ground was taken from Hapke (1981) :

$$
f\left(\theta_{a}, \phi_{r} ; \theta_{r}, \phi_{r}\right) \quad \frac{\omega}{i \pi \mu_{r} \mid \mu_{r}}\left[\{1 \mid B(g)\} P(g)+I\left(\mu_{r}\right) H\left(\mu_{r}\right) \cdots 1\right],
$$

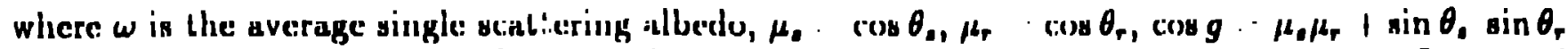
$\cos \left(\phi_{r} \phi_{1}\right), B(g) \ldots B B_{0} /\left[1+h^{1} \tan _{.} / 2\right)\left|, B_{n}=S(0) /(\omega / P(0)), P(g): 1\right| b \cos g+c\left|\left(3 \cos ^{2} g-1\right) / 2\right|$ and $I(x) \cdot(1+2 x) /\left(1+2|1-w|^{1 / 2} x\right)$. 'The BRDF parameters chosen were: $w \cdot 0.57, S(0)$. $0.48, h \quad(0.21, b-(0.86$ and $c *$ (0.7. 'The BRDF of vegelated surface was laken from a parametric: model by Pinty et al (1990) with the same notation as above where not listed.

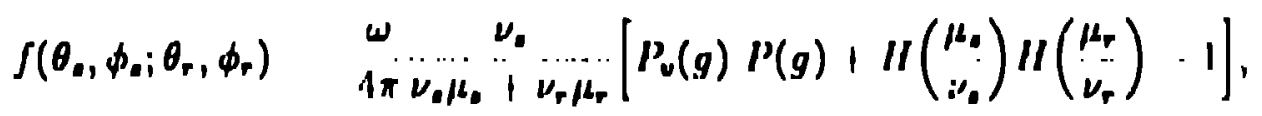

where: $\nu_{a}$ and $\nu_{r}$ describe the lenf orientation distribution for the illumination and observation angles which depend on at parnmeter $x_{1}$ with range: $(1) 1: x_{1}:(1.6), l^{\prime}(g)$ is the leal neat cering phase function which in the Ifenyey-(ircenotein function in eq (5), ihe function $P_{v}(g)$ depends in the variuble (; $\left|\operatorname{tnn} !^{2} \theta_{1}+\tan ^{2} \theta_{r} \quad 2 \operatorname{lnn} \theta_{1} \tan \theta_{r} \cos \left(\phi_{g} \phi_{r}\right)\right|^{1 / 2}$, the radius of sun Ilrckur in $|m|$ and $\Lambda$ the lenf area density in $\mid m^{2} m \mu$. We selected the following ('anopy parameters : $\omega \quad 0.8,(6) \quad 0.1, \Lambda \quad\left(0.01, r \quad 1 ., x_{1} \quad(1.2\right.$.

Fior the water surface we assumed the HRLI)F to be a Henyey (ireensteiu phase function $\int\left(\theta_{0}, \phi_{a} ; \theta_{r}, \phi_{r}\right) \quad \rho_{\text {wator }} l(g)$ with the forward penk nligned with the spectular reflectance direction

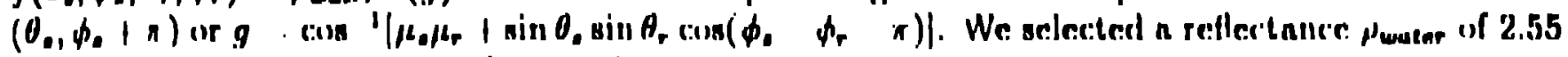
$\%$ and nil nsymmetry factur' of $\theta$ (1.9). 
In Figure 2 we show the point spread functions for (a) bare soil, (b) vegetation and (c) water. Note that the PSF's are asymmetric and that the PSF for water has a ridge in the specular reflection direction (left side) of the sun which has a zenith angle of $30^{\circ}$.

In Figure 3 we show a simulated scene containing some rectangular vegetated surfaces (light gray) surrounded by bare soil (dark gray) and a lake (black) in the middle. The simulated scene measures $3 \mathrm{~km}$ by $3 \mathrm{~km}$ and is viewed from the below with a zenith angle of $60^{\circ}$. The sun light is incident from above with a zenith angle of $30^{\circ}$. To make faint radiance changes more visible we show the histogram equalized radiance image. One can see the effect of the specular reflection of the sunlight into the atmosphere on the radiance in the field at the top of image (b). The edges of the fields closer to the observer appear darker than the edges away from the observer. Near the lower edge of the lake, the radiance decreases while it increases at the upper edge due to the increased scattering above the water surface.

\section{Conclusions}

The extended radiosity method has bee. used tc compute point spread functions for a layered almosphere above a heterogeneous ground cover The adjacency blurring effect was simulated for a scene containing vegetated, bare soil and water surfaces.

\section{References}

[1] C.C. Borcl, S.A.W. Gerstl, "Simuiation of partially obscured scenes using the ratiosity method", SPIE' Vol. 1486, pp.271-277, A pril 1991.

[2] C.C. Borel, S.A.W. Gerstl, "Atenospheric corrections using the volume radiosily method". Proc. Int. Geoscience and Remote Sensing Symposium, May 26.29, 1992, Ilouston, Texas, 1992.

[3] D.J. Diner and J.V Martonchik, "Atmospheric trangmittance from spacecraft using nultiple: view angle imagery", Applicd (Opties, :24:21, pp.3503-3511, 1985.

[4] 13. Hapke, "Hidirectional reflectance speectroscopy: 1. Lheory", J. Grophys. Re.s., Vol. 86, No. B4, pp. 3039-3054, April, 1981.

[5] Y.J. Kaufrnan, "Almospheric ellects on remonte sensing of surface reflectance", SPIl' proceedings, 175:20-33, 1984.

(6) K.-N. liol', An introduction to almospheric radiation. Academic P'rens, ()rlando, lil, I:880.

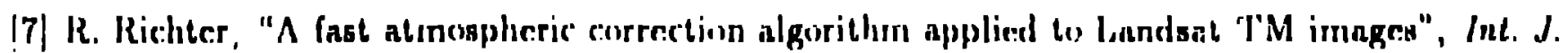
iremote Se:nstng, 11, pp. 159.1(6i, 19901).

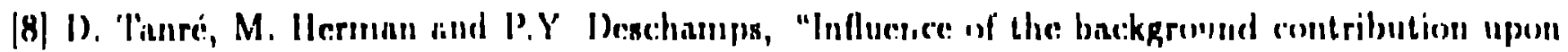

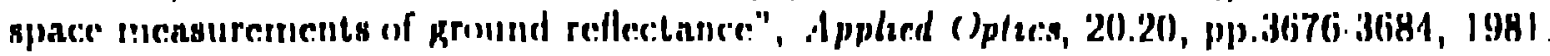

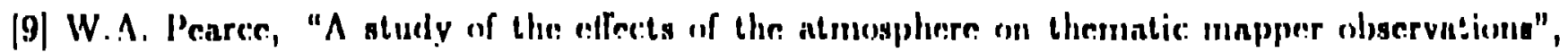

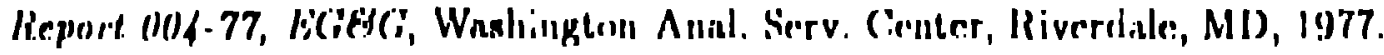

|10| 13. Pinty, M. Veratraete and R. I)ickinson, "A physicnl medel of the bidire:tennal reflectonce of

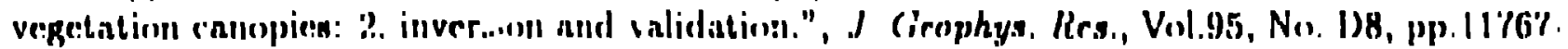
$11775,1990)$. 


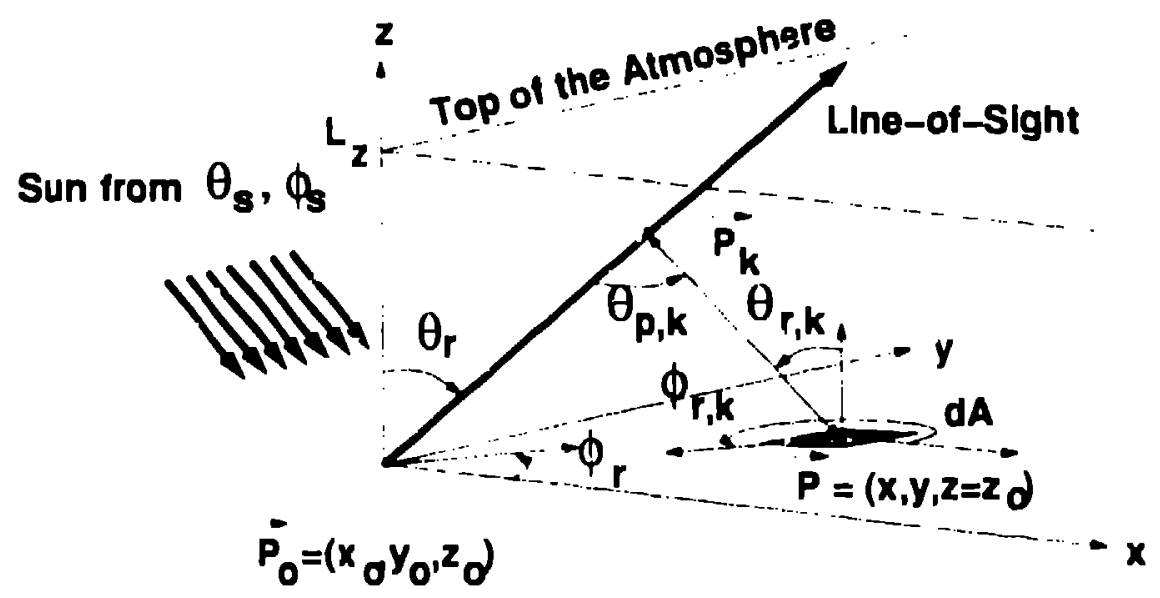

Figure 1 Geometry for computing the point spread function.
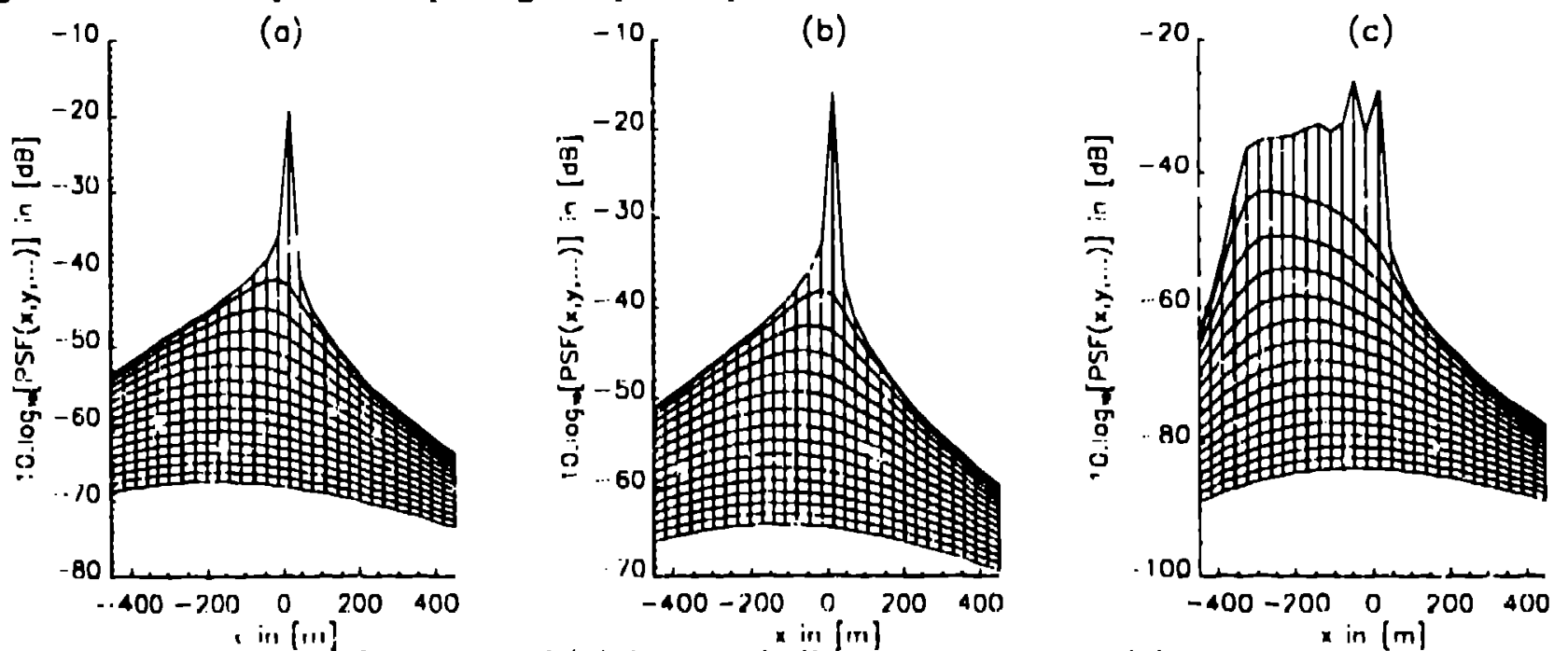

Pigure 2 Point spread functions of (a) bire soil, (b) vegetation and (c) water with the z-axis in lugarithmic scale and the $y$-axis points into the paper.

(a)

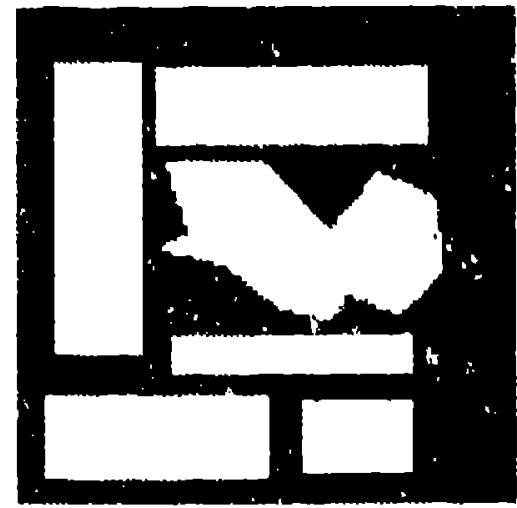

(b)

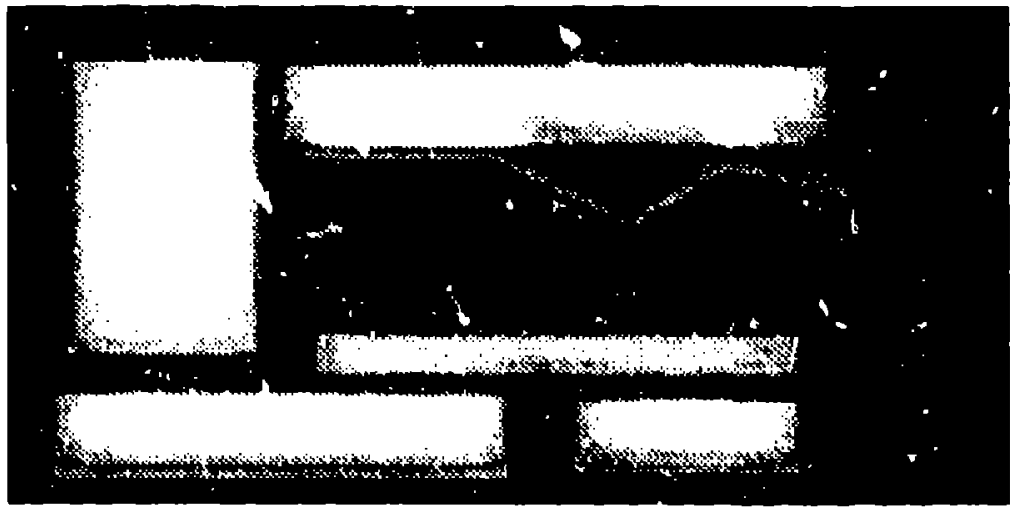

l'igure 3 Simulated acene from (a) nbove and (b) viewed through ntmosphere from the below at (b0) view renith angle and illuminated from above at $30^{\circ}$ sun zenith angle. 